\title{
A Diagnostic Pitfall in a Patient with Acute Abdominal Pain: Congenital Hydronephrosis/Hydroureter Misdiagnosed as Hydrosalpinx
}

\author{
Tharmmambal Balakrishnan ${ }^{*}$, Deepak Rama Narayana ${ }^{2}$ \\ ${ }^{1}$ Department of Internal Medicine, Singapore General Hospital, Singapore City, Singapore \\ ${ }^{2}$ Department of Laboratory Medicine, Khoo Teck Puat Hospital, Singapore City, Singapore \\ Email: ${ }^{*}$ tharmmamba.b@sgh.com.sg, Ramanarayana.deepak@alexandrahealth.com.sg
}

Received 2 April 2014; revised 12 May 2014; accepted 25 June 2014

Copyright (C) 2014 by authors and OALib.

This work is licensed under the Creative Commons Attribution International License (CC BY). http://creativecommons.org/licenses/by/4.0/

(c) (i) Open Access

\begin{abstract}
Clinical manifestation or incidental finding of congenital kidney anomalies are more often discovered during pregnancy. Even if the acute presentation (in the antenatal period, as in this case) precludes a full diagnostic workup, assiduous follow-up and investigations in the postnatal period will resolve the initial uncertainty. This is not a new phenomenon based on literature and urologic differential diagnoses such as an ectopic urinary system which should always be entertained and excluded prior to making the diagnosis of an adnexal mass. We present a case of 33-year-old woman with acute abdominal pain and a previous obstetric (mis)diagnosis of hydrosalpinx (based on ultrasonography) made 8 years before presenting to the emergency department with acute lower abdominal pain, fever, chills and rigors. Current investigations revealed congenital abnormal kidneys bilaterally, with ectopic left kidney and grossly infected hydronephrosis, which was likely the fluid-filled mass initially mistaken for a hydrosalpinx. She then underwent left nephrostomy and later had simple left nephrectomy. This case highlights the importance of making an accurate diagnosis and arranging careful follow-up of the female patient presenting with a "cystic" mass of the pelvic region during pregnancy.
\end{abstract}

\section{Keywords}

Congenital Malformation of Kidney, Ectopic Kidney, Hydrosalpinx, Hydronephrosis, Malrotated Kidney

Subject Areas: Gynecology \& Obstetrics, Urology, Women's Health

\footnotetext{
*Corresponding author.
}

How to cite this paper: Balakrishnan, T. and Narayana, D.R. (2014) A Diagnostic Pitfall in a Patient with Acute Abdominal Pain: Congenital Hydronephrosis/Hydroureter Misdiagnosed as Hydrosalpinx. Open Access Library Journal, 1: e701. 


\section{Introduction}

Lower abdominal pain is common among woman and to determine the aetiology; it requires investigation based on clinical suspicion and may need assessment of all systems involved anatomically [1]. Although uncommon, it is important to be aware as pregnancy may reveal many congenital abnormalities, which comes to attention as it may be the initial contact that requires imaging for most women [2].

\section{Case Report}

The patient who was then 25 years old initially saw a specialist 8 years before the emergent presentation. At the time, the patient was gravid with her first and only child and had no significant past medical history and had no significant past medical history. An antenatal ultrasound performed for reduced fetal movements at term showed a right sided fluid-filled mass measuring approximately $11 \mathrm{~cm}$ in greatest diameter. The mass was labelled as "hydrosalpinx". As it was felt that the mass might obstruct labour, it was drained transvaginally with $500 \mathrm{ml}$ of "straw-coloured fluid” being aspirated. Cytology of the fluid was unremarkable. The patient proceeded to have an uneventful normal vaginal delivery at term 10 days after the drainage procedure. No further follow-up of the cystic mass was arranged (Figure 1 ).

The patient remained quite well in the intervening eight years until her acute presentation to the emergency department with severe pain in her left iliac fossa and suprapubic region associated with urgency and frequency of micturition. The patient also had chills and rigors but no documented fever at home. She denied any vaginal discharge. She was not on hormonal contraception, had a regular menstrual cycle, and had never had any intra-uterine devices inserted.

On examination in the emergency department, the patient was febrile with a temperature of $38.6^{\circ} \mathrm{C}$, blood pressure 116/66 mmHg, pulse rate of $130 \mathrm{bpm}$ and a respiratory rate of 19 per minute. Abdominal examination revealed suprapubic and left iliac fossa rebound and percussion tenderness. Abdominal palpation revealed a mass (dull to percussion) present on the right iliac fossa and approximating the size of a 20-week uterus. The renal punch was negative bilaterally.

A complete blood count revealed mild leucocytosis $\left(11.30 \times 10^{9} / \mathrm{L}\right)$ with $88 \%$ neutrophil predominance. She also had mild microcytic anemia with a hemoglobin of $10.8 \mathrm{~g} / \mathrm{dL}$ with Mean Cell Volume (MCV) of $74.6 \mathrm{fL}$. Biochemical investigations included a normal serum creatinine of $69 \mathrm{umol} / \mathrm{L}$ with raised inflammatory markers such as serum C-reactive protein of $288 \mathrm{mg} / \mathrm{L}$ and procalcitonin of $1.5 \mathrm{ug} / \mathrm{L}$. Urine microscopy showed hematuria and pyuria with $>2000$ red cells and $>2000$ white cells per $u L$. She had a negative urine pregnancy test. The differential diagnosis to be considered here were pyosalpinx, torsion of hydrosalpinx, pyelonephritis, cystitis or pelvic inflammatory disease.

Patient was admitted and blood cultures were taken. A CT scan of the abdomen and pelvis was done, showing congenitally malformed kidneys. The left kidney was low lying in the midline with gross hydronephrosis $(15 \mathrm{~cm}$
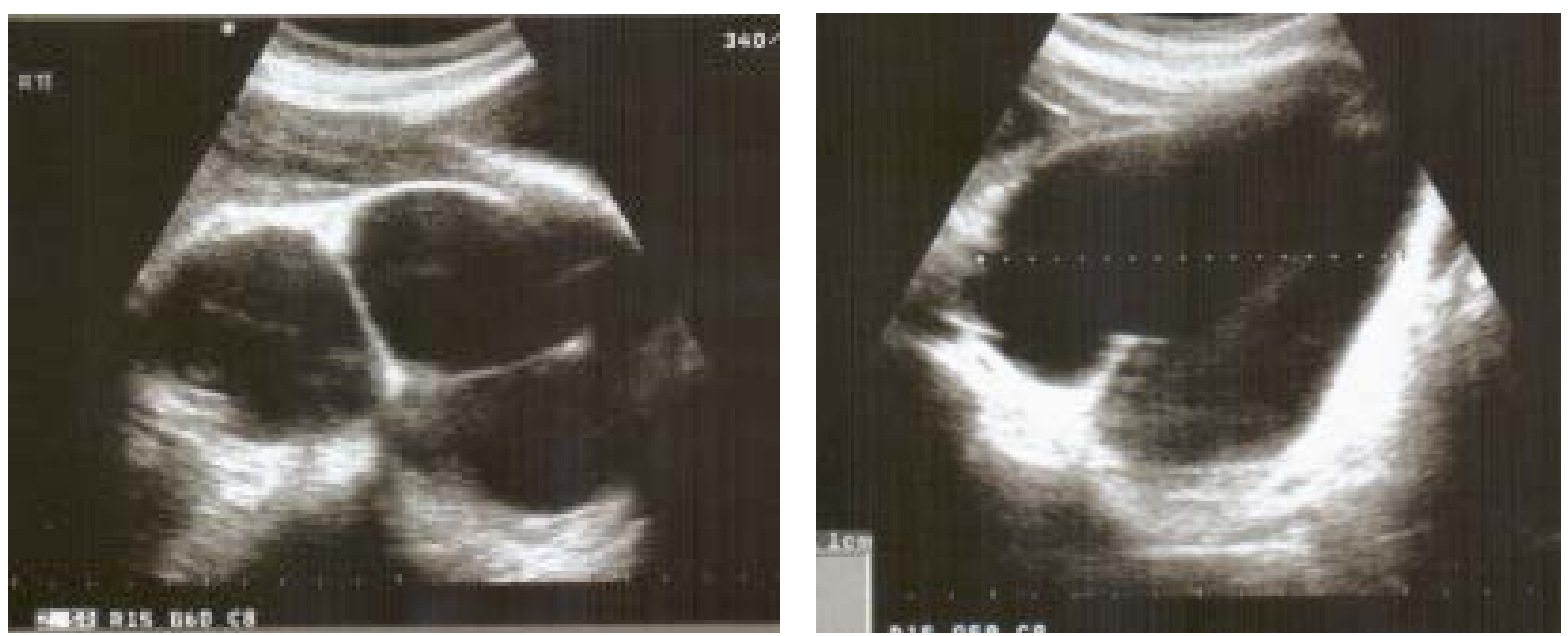

Figure 1. Abdominal and pelvic ultrasound images at 24 weeks gestation both cross section and sagittal views demonstrating “cystic mass". 
bipolar length, $11.5 \mathrm{~cm} \times 13.5 \mathrm{~cm}$ in cross section) and angulated pelviureteric and vesicoureteric junctions. There was no calyceal fluid level or presence of stones noted and this obstruction was noted to be chronic. No perinephric stranding was noted. The right kidney had an abnormal orientation with right renal pelvis facing laterally and demonstrated duplex system. There was no hydroureter or hydronephrosis on right kidney. The reproductive system was normal with no hydrosalpinx noted (Figure 2 and Figure 3).

Intravenous antibiotic therapy was begun however, in view of her previous obstetric history, a gynaecology consult was obtained in the emergency department and patient underwent a bedside transvaginal and transabdominal ultrasonography. The scan showed a right-sided loculated, septated, cystic lesion, separate from the ovary, measuring $13.2 \mathrm{~cm}$ in greatest diameter. As there was an infected hydronephrotic collection serving as a source for persistent sepsis, the patient underwent left nephrostomy drainage. The patient's symptoms were relieved and her temperature quickly settled with continued antibiotic therapy. The patient was discharged on con

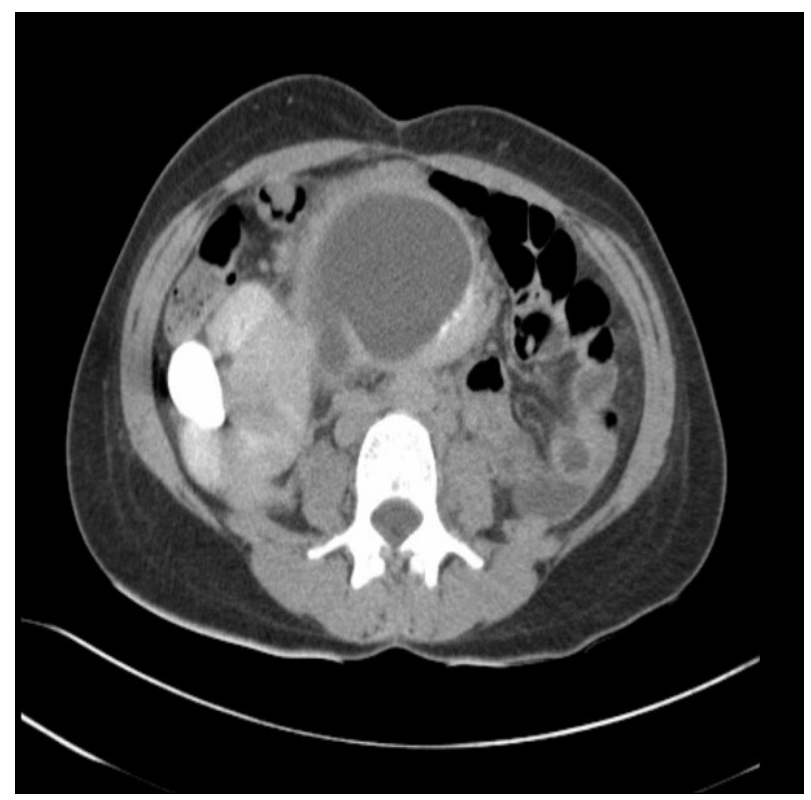

Figure 2. Axial CT scan view showing malrotated right kidney.

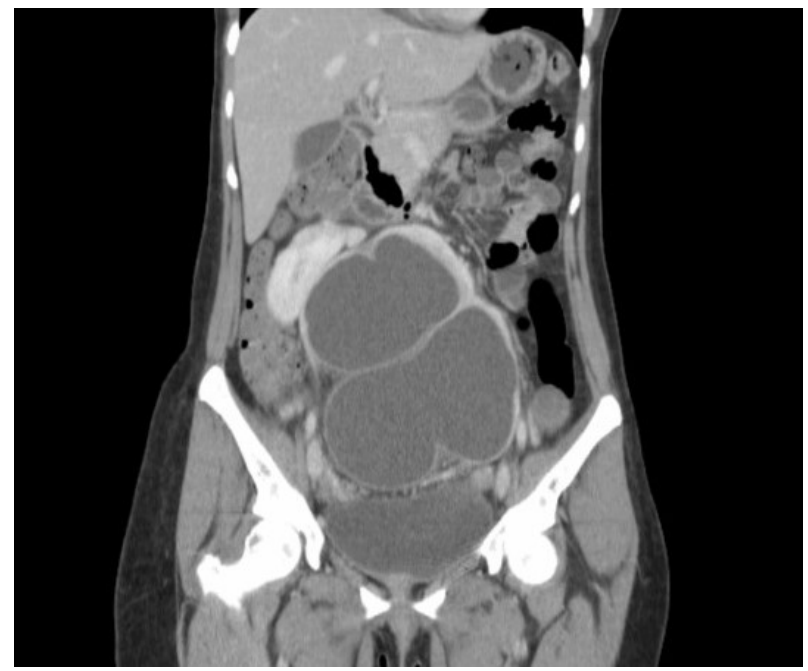

Figure 3. Coronal CT scan view showing ectopic (pelvic) and gross hydronephrosis of left kidney located midline extending into right side. 
tinuous nephrostomy drainage and outpatient intravenous Ceftriaxone therapy. She underwent an outpatient anterograde nephrostogram to better visualise the abnormal anatomy. As it was felt that there was insufficient functioning renal tissue to be salvaged in the dilated left renal system, and in order to prevent recurrence, she underwent a left-sided simple nephrectomy. Patient has remained stable postoperatively and was well on outpatient follow up visits subsequently.

\section{Discussion}

A presentation of acute lower abdominal pain in a woman (pregnant or otherwise) of reproductive age may reasonably lead to differential diagnosis of adnexal pathology including torsion of hydrosalpinx and pyosalpinx. The latter may also be associated with clear signs of systemic sepsis, including fever and neutrophilia. A hydronephrotic kidney in an anatomically unexpected location and orientation can easily be mistaken for one of these gynaecological emergencies [1]. The incidence of ectopic kidney among pregnant women varies between 0.2 per 1000 and 1 per 1000 [2], making the condition sufficiently uncommon that it may not immediately be considered as a differential diagnosis.

A case of ectopic hydronephrotic kidney masquerading as an ovarian cyst during pregnancy been reported by Neena et al. in 2000; this case involved a young woman presenting with recurrent severe pain in lower abdomen. The ultrasonography done at 26 weeks gestation revealed a "cyst" which was removed by emergency laparotomy. The histology of the "cyst" revealed an end stage hydronephrotic kidney [3]. So the initial presentation of ectopic kidney and associated abnormalities during pregnancy is not without precedent in the literature.

Ectopic kidney is often associated with other abnormalities such as agenesis of the opposite kidney, vascular malformation and genital anomalies [4]. Most renal anomalies are incidental findings [5] and most ectopic kidneys are clinically asymptomatic and they are not any more susceptible to disease than normally positioned kidneys; however, they may become complicated by the development of hydronephrosis or urinary calculus formation [6]. Even an otherwise uncomplicated ectopic kidney may pose a diagnostic conundrum, as the abnormal position of the organ may result in a pattern of direct and referred pain that is atypical for colic and maybe misdiagnosed as acute appendicitis or pelvic inflammatory disease in women [7].

Hertzberg et al has described about colour doppler flow which can show the pulsation or peristalsis in the dilated segment favors a dilated ureter or fluid filled loop of bowel over the dilated fallopian tube and this is only true if it is a functioning kidney [8]-[10]. Colour doppler ultrasound in pregnancies allows functional assessment of genitourinary tract with better diagnostic accuracy in detecting obstructive hydronephrosis [11]. Magnetic resonance imaging (MRI) is recommended in evaluating hydronephrosis in pregnancy [12]. Diagnosis of hydrosalpinx can be made with presence of tubular mass with waist sign or identification of small round projections on ultrasound [13]. Adnexal mass is not uncommon in pregnancy and usual presentation is with pelvic pain and MRI is a suggested modality for investigation of any adnexal mass including hydrosalpinx that is often associated with underlying structural abnormality [14] [15].

Physiological hydronephrosis occurs in $90 \%$ of pregnancies and is the commonest maternal urological abnormalities but less than $3 \%$ are symptomatic [12]. The likely possibility in our patient was a physiological hydronephrosis which was noted to be a cystic mass at 24 weeks or even hydroureter that was thought to be hydrosalpinx at term and it resolved postpartum but re-emerged as acute obstruction and associated septicaemia.

Establishing the correct diagnosis of congenital urological abnormalities is important so that correctable conditions can be addressed, and future complications pre-empted. In relevance to the case under discussion, it is understandable that a misdiagnosis of adnexal mass was made on initial presentation during pregnancy. However, close follow-up and further investigations during or following delivery should have been arranged. It is conceivable that the complications suffered by this patient may have been avoided with better follow-up. Presentation with recurrent urinary tract infections and chronic pelvic pain in a woman should trigger an evaluation for congenital anomalies of the urinary system.

\section{Conclusion}

In summary, although uncommon diagnosis of hydrosalpinx should be evaluated further with following up scans as it may not cause any clinical symptoms at that point and potentially present with unrelated pathology due to sonographic investigation that mimics other condition. 


\section{Conflict of Interest}

None.

\section{References}

[1] Hernandez-Rey, A.E., Vitensen, J. and McGovern, P.G. (2007) Duplicated Ectopic Hydroureter Presenting as Hydrosalpinx, with Chronic Pelvic Pain and Recurrent Urinary Tract Infections. Fertility and Sterility, 88, 1677e1-e3.

[2] Bergqvist, A. (1965) Ectopic Kidney as Complication of Pregnancy and Labor. Acta Obstetrica et Gynecologi ca Scandinavica, 44, 289-303. http://dx.doi.org/10.3109/00016346509155866

[3] Neena M. (2001) Ectopic Hydronephrotic Kidney Masquerading as an Ovarian Cyst during Pregnancy. European Journal of Obstetris \& Gynecology and Reproductive Biology, 97, 239-240. http://dx.doi.org/10.1016/S0301-2115(00)00516-9

[4] Rascher, W. and Rosch, W.H. (2005) Congenital Abnormalities of the Urinary Tract. Oxford Textbook of Clinical Nephrology. Oxford University Press, Inc., Oxford, 1402-1412.

[5] Kemper, M.J. and Muller-Wiefel, D.E. (2001) Renal Function in Congenital Anomalies of the Kidney and Urinary Tract. Current Opinion in Urology, 11, 571-575. http://dx.doi.org/10.1097/00042307-200111000-00003

[6] Campbell, M.F., Wein, A.J. and Kavoussi, L.R. (2007) Campbell-Walsh Urology. 9th Edition, Saunders Elsevier, Philadelphia, 3279.

[7] Kang, I.J., Lee, S.H., Lee, S.M., Lim, C.H., Yoon, J.H., Kim, T.W., Seo, S.M., Kim, B.S. and Chang, Y.S. (2005) A Case of Congenital Single Ectopic Kidney in Pelvis of Patients with Proteinuria. Korean Journal of Nephrology, 24, 137-140.

[8] Hertzberg, B.S., Kliewer, M.A., Leder, R.A. and Carroll, B.A. (1997) Primary Megaureter Mimicking Hydrosalphinx on Ultrasonography. Journal of Ultrasound in Medicine, 16, 441-444.

[9] Kim, J.M., Hong, J.S., Yoon, J.B., Cho, J.H. and Joo, K.H. (2003) Marked Hydroureter Misdiagnosed as Hydrosapinx by Ultrasound. Journal of the American Association of Gynecologic Laparoscopists, 10. http://dx.doi.org/10.1016/S1074-3804(05)60141-7

[10] Zalel, Y., Soriano, D., Lipitz, S., Mashiach, S. and Achiron, R. (2000) Contribution of Color Doppler Flow to the Ultrasonographic Diagnosis of Tubal Abnormalities. Journal of Ultrasound in Medicine, 19, 645-649.

[11] Pepe, F. and Color, P.P. (2013) Doppler Ultrasound (CDU) in the Diagnosis of Obstructive Hydronephrosis in Pregnant Women. Archives of Gynecology and Obstetrics, 288, 489-493. http://dx.doi.org/10.1007/s00404-013-2768-1

[12] Fiadjoe, P., Kannan, K. and Rane, A. (2010) Maternal Urological Problems in Pregnancy. European Journal of Obstetrics \& Gynecology and Reproductive Biology, 152, 13-17. http://dx.doi.org/10.1016/j.ejogrb.2010.04.013

[13] Patel, M.D., Acord, D.L. and Young, S.W. (2006) Likelihood Ratio of Sonographic Findings in Discriminating Hydrosalpinx from Other Adnexal Masses. American Journal of Roentgenology, 86, 1033-1038. http://dx.doi.org/10.2214/AJR.05.0091

[14] Masselli, G., Brunelli, R., Casciani, E., Polettini, E., Bertini, L., Laghi, F., Anceschi, M. and Gualdi, G. (2011) Acute Abdominal and Pelvic Pain in Pregnancy: MR Imaging as a Valuable Adjunct to Ultrasound? Abdominal Imaging, 36, 596-603. http://dx.doi.org/10.1007/s00261-010-9654-0

[15] Kim, M.Y., Rha, S.E., Oh, S.N., Jung, S.E., Lee, Y.J., Kim, Y.S., Byun, J.Y., Lee, A. and Kim, M.R. (2009) MR Imaging Findings of Hydrosalpinx: A Comprehensive Review. Radiographics, 29, 495-507. 